10

\title{
Акустооптическое управление энергетическим профилем лазерного излучения
}

\author{
(c) С.Н. Антонов, А.Л. Филатов
}

Институт радиотехники и электроники им. В.А. Котельникова РАН, 141190 Фрязино, Московская обл., Россия

e-mail: olga-ant@ya.ru

(Поступило в Редакцию 25 марта 2017 г. В окончательной редакции 17 мая 2017 г.)

Разработан акустооптический шепер (shaper) - прибор, предназначенный для преобразования гауссова лазерного излучения в вид, близкий к прямоугольному. В отличие от линзовых приборов акустооптический позволяет модифицировать форму выходного луча при фиксированной оптической конфигурации путем изменения радиоуправления с высоким быстродействием (около $10 \mu \mathrm{s}$ ). Основой метода является многолучевая высокоэффективная брэгговская дифракция, при которой реализуется угловое перекрытие дифракционных порядков. Показано, что с эффективностью не менее $80 \%$ можно производить преобразование формы лазерного луча не только в прямоугольный вид, но и в более сложный. Эксперименты произведены на базе кристалла $\mathrm{TeO}_{2}$.

DOI: 10.21883/JTF.2018.01.45489.2266

\section{Введение}

Акустооптика $(\mathrm{AO})$ - воздействие акустически сформированных дифракционных решеток на проходящий свет. Изучались разнообразные возможности АО: анализаторы радиосигналов, приборы спектральной обработки изображений, оптические процессоры и др. [1]. Однако многосторонняя технологическая революция оставила только направления, связанные с управлением параметрами лазерного излучения (ЛИ): дефлекторы (сканеры) и модуляторы (сдвигатели частоты) света.

Ренессанс АO связан с революцией в лазерной технике - созданием мощных (более десятков $\mathrm{kW}$ ), компактных, с выдающимся КПД (вплоть до 50\%) волоконных лазеров. Тут и проявились особенности АО - управление очень интенсивным лазерным излучением (более $1 \mathrm{~kW} / \mathrm{cm}^{2}$ ), достаточно высокое быстродействие (вплоть до десятков ns), отсутствие механически перемещаемых элементов, малые вносимые световые потери (до единиц процентов), практически неограниченный ресурс работы [2].

Акустооптические модуляторы формируют „гигантскийс импульс в импульсных лазерах [3]. Дефлекторы (сканеры) применяются в системах обработки материалов, устройствах лазерного вывода изображений, переключателях оптических каналов при оптической передаче данных, устройствах навигации и позиционирования элементов конструкций.

В настоящей работе сообщаем о новом применении $\mathrm{AO}$ - управление энергооптического профиля (ЭП) лазерного излучения.

\section{Энергетический профиль лазерного излучения}

Решаемая проблема связана с тем, что в системах лазерной обработки материалов: резка, маркировка, сварка и т.д. нормальное (гауссово) угловое распределение интенсивности ЛИ не является оптимальным. На рис. 1 показано нормированное гауссово угловое распределение - непрерывная кривая 1 и порог - линия 2, ниже требуемого воздействия (испарение, горение) на материал не происходит.

В качестве примера энергетический порог воздействия равен уровню $I_{0}=0.9$. Тогда отношение площади заштрихованной части (полезно используемой мощности) ко всей площади под кривой (полной мощности) составляет $\approx 35 \%$, и более $60 \%$ потока энергии не используется. Для эффективного использования мощности ЛИ целесообразен свет с ЭП, близким к Побразному, а в ряде случаев и более сложному. Так, например, в системах спекание металлического порошка в 3-D принтерах более выгоден не плоский, а неровный „верх“ распределения ЛИ. На рис. 2 показан один из промышленных приборов такого типа - оптический шейпер (shaper) модели $\pi$ Shaper 12-12-355. Прибор со-



Рис. 1. Классическая форма гауссового распределения. Энергетическая часть заштрихована. $\Theta-$ нормированные углы, $I$ - относительная интенсивность света. 


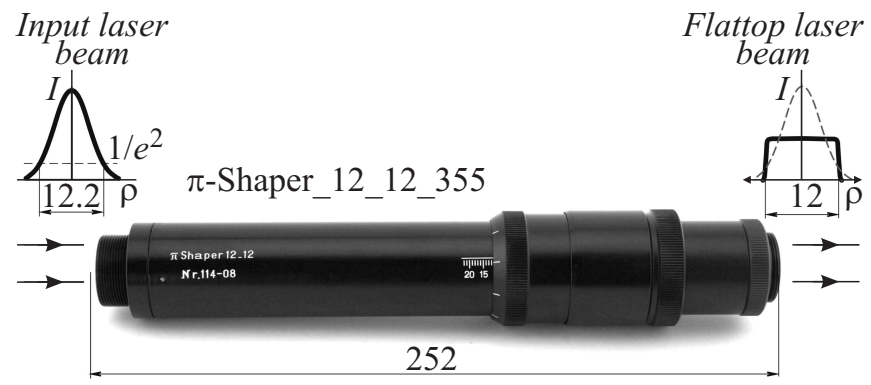

Рис. 2. Внешний вид линзового шейпера.

стоит из несколько специально изготовленных линз. Исходно гауссовый лазерный луч трансформируется в излучение с неизменяемым (для контренной модели прибора) ЭП, близким к прямоугольному.

Настоящая работа посвящена АО-шейперу, позволяющему быстропеременно изменять параметры ЭП выходного луча при фиксированной оптической конфигурации - положению АО прибора и параметрах входного луча.

\section{Акустооптическая высокоэффективная многолучевая дифракция}

Формирование ЭП базируется на недавно обнаруженном АО-эффекте [4-7] - многолучевой брэгговской АОдифракции, который обеспечивает высокоэффективное (до 100\%) расщепление одного входного лазерного луча на несколько лучей с одинаковой интенсивностью. До работы [4] полагалось, что такое расщепление возможно только при малой суммарной эффективности в отклоненных лучах, что поясняется рис. 3.

Входной лазерный луч $k_{0-}$,разбивается“ на дифракционные порядки при подаче на пьезопреобразователь нескольких независимых частот. Если суммарная мощность сигнала соответствует индексу АО фазовой модуляции, близкой к $\pi$, то в общем случае

- возникнут дифракционные порядки (рабочие) $k_{1}$, связанные с наличием вводимых акустических волн,

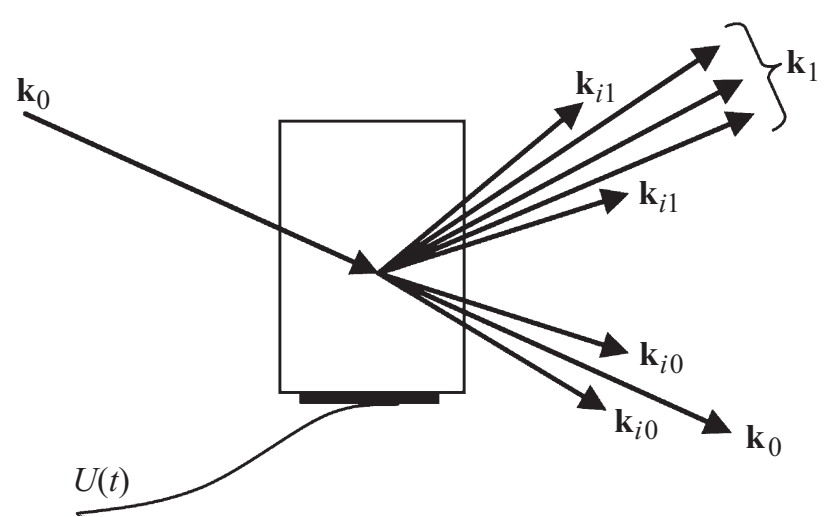

Рис. 3. Диаграмма образования основных и паразитных дифракционных лучей при многочастотной АО-дифракции.
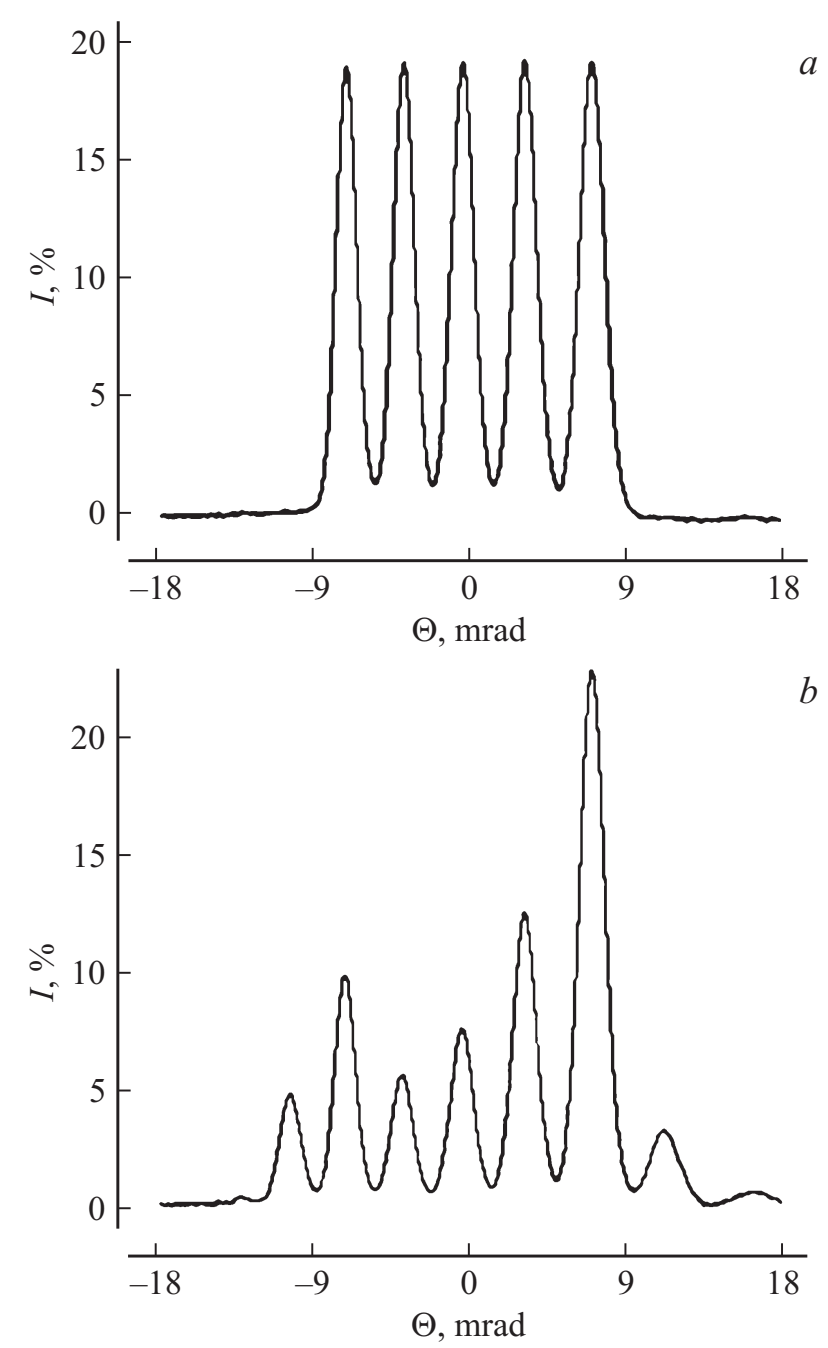

Рис. 4. Экспериментальные угловые распределения световой мощности: $a-$ с оптимальными фазовыми соотношениями радиосигнала, $b-$ со случайными фазами сигналов.

- помимо этого, в силу интермодуляционных эффектов в дифрагированном поле и вблизи нулевого порядка возникнут дополнительные порядки $k_{i 1}$ и $k_{i 0}$ соответственно,

- интенсивность каждого из основных лучей будет нелинейным (сложным) образом зависеть от количества и мощностей вводимых электрических сигналов.

Новая особенность АО проявляется, когда акустический сигнал подобен частотно-модулированному, т.е. представляет собой сумму синусоидальных сигналов, эквидистантных по частоте и связанных по фазе и амплитуде такими соотношениями, которые минимизируют амплитудную модуляцию сигнала [5]:

$$
\begin{gathered}
U(t)=a(t) \exp (i \omega t), a(t)=\Sigma_{n}\left(A_{n} \exp \left(i n \Omega t+i \varphi_{n}\right)\right) \\
|a(t)| \approx \text { const. }
\end{gathered}
$$

Здесь $U(t)$ - электрический сигнал, подаваемый на пьезопреобразователь АО-модулятора, $\omega$ - его центральная частота, $i$ - мнимая единица, $a(t)-$ комплексная 
огибающая сигнала, $A_{n}$ - действительные амплитуды отдельных частотных компонент (их число - $n$ ), $\varphi_{n}-$ начальные фазы частотных компонент, $\Omega$ - шаг частот спектра сигнала. Важно, чтобы период модуляции был меньше времени прохода звука через апертуру света. Кроме того, интенсивность каждого из основных лучей будет сложным, нелинейным образом зависеть от количества и мощностей всех частотных компонент сигнала, и при равных мощностях компонент интенсивности лучей будут неодинаковы. Найденная новая особенность АО-взаимодействия проявляется тогда, когда акустический сигнал подобен частотно-модулированному, т.е. представляет собой сумму синусоидальных сигналов, эквидистантных по частоте и связанных по фазе и амплитуде такими соотношениями, которые минимизируют амплитудную модуляцию суммарного сигнала.

Выполнение условия (1) обеспечивает практически полное исчезновение интермодуляционных „паразитных“ дифракционных порядков и отклонение всей световой мощности в основной дифракционный порядок $k_{1}$. При этом каждая угловая компонента порядка связана только с соответствующим электрическим сигналом. При величине $\Omega$, превышающей некоторое значение, дифракционное поле состоит из отдельных неперекрывающихся световых лучей.

Рис. 4 проиллюстрирует эффект многолучевой дифракции. На осциллограммах показан угловой спектр света на выходе АО-модулятора при подаче на него суммы пяти синусоидальных электрических сигналов. Здесь ось $X$ - угловая координата, ось $Y$ - интенсивность световых лучей. Рис. 4, $a$ - фазовые и амплитудные соотношения между сигналами подобраны так, чтобы суммарный акустический отклик был подобен частотномодулированному. В результате дифрагированное поле состоит из пяти лучей равной интенсивности при суммарной эффективности дифракции, близкой к 100\%. Рис. $4, b$ соответствует случайному фазовому соотношению между сигналами, но с теми же амплитудами.

\section{П-образный энергетический профиль, практические результаты}

Многолучевая АО-дифракция явилась базой для создания АО-шейпера. Решение заключается в следующем. Из (1) следует, что уменьшение $\Omega$ будет приводить к перекрытию соседних лучей и образованию единого луча. Степень перекрытия определяется величиной $\Omega$. Таким образом, количество лучей и степень перекрытия определяют угловую ширину итогового луча, а угол наклона „краев“ луча связан с параметрами исходного гауссовога пучка. Величины амплитуд $A_{n}$ и значения фаз $\varphi_{n}$ определяют форму итогового луча. Заметим, что П-образность - это частный случай, что будет показано ниже.

Отметим существенный факт. Термин „единый луч“ корректен лишь в том случае, если не учитывать интерференцию (биения), возникающую в областях перекрытия соседних лучей. Частота биений равна разности

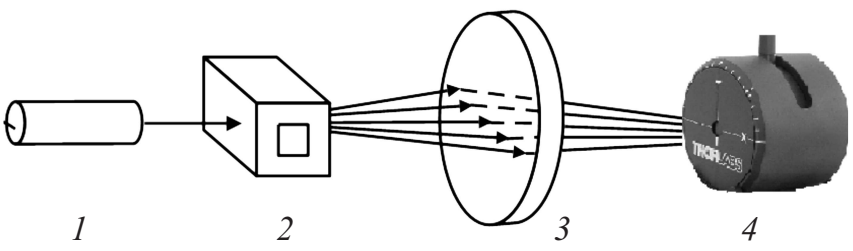

Рис. 5. Оптическая схема измерительной установки.
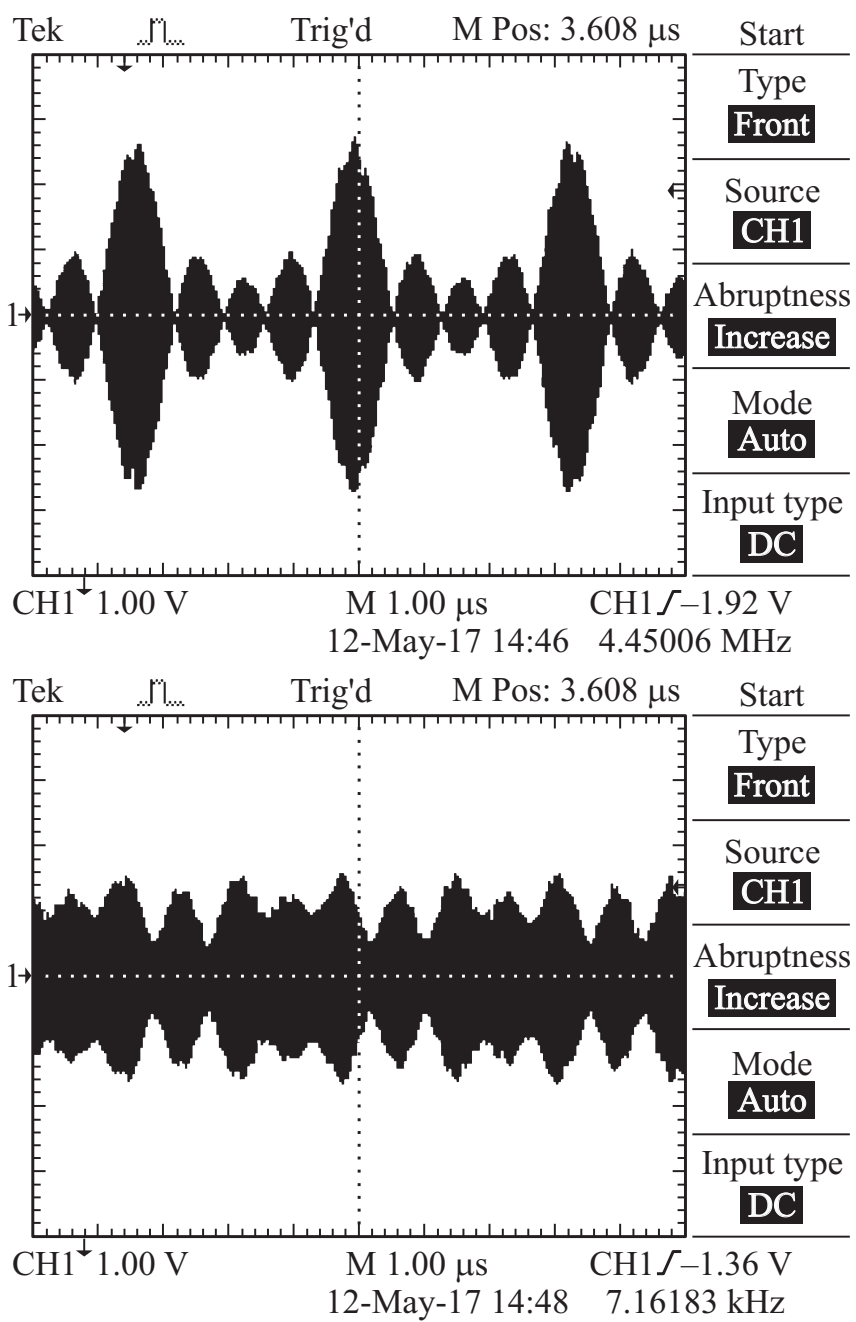

Рис. 6. Электронная фотография сигналов на входе АОдефлектора: $a-$ произвольное фазовое соотношение между частотными компонентами, $b-$ подобранные фазовые соотношения.

частот ближайших электрических сигналов (в условиях эксперимента это $0.3 \mathrm{MHz}$ ). В большинстве практических случаев, связанных с лазерной обработкой материалов, смещение луча или материала „от точки к точке“ не превышает десятка миллисекунд. При таком усреднении термин единый луч вполне правомерен.

Принципиальная оптическая схема установки приведена на рис. 5. Базовый инструмент - измеритель профиля оптического излучения модели THORLABS 

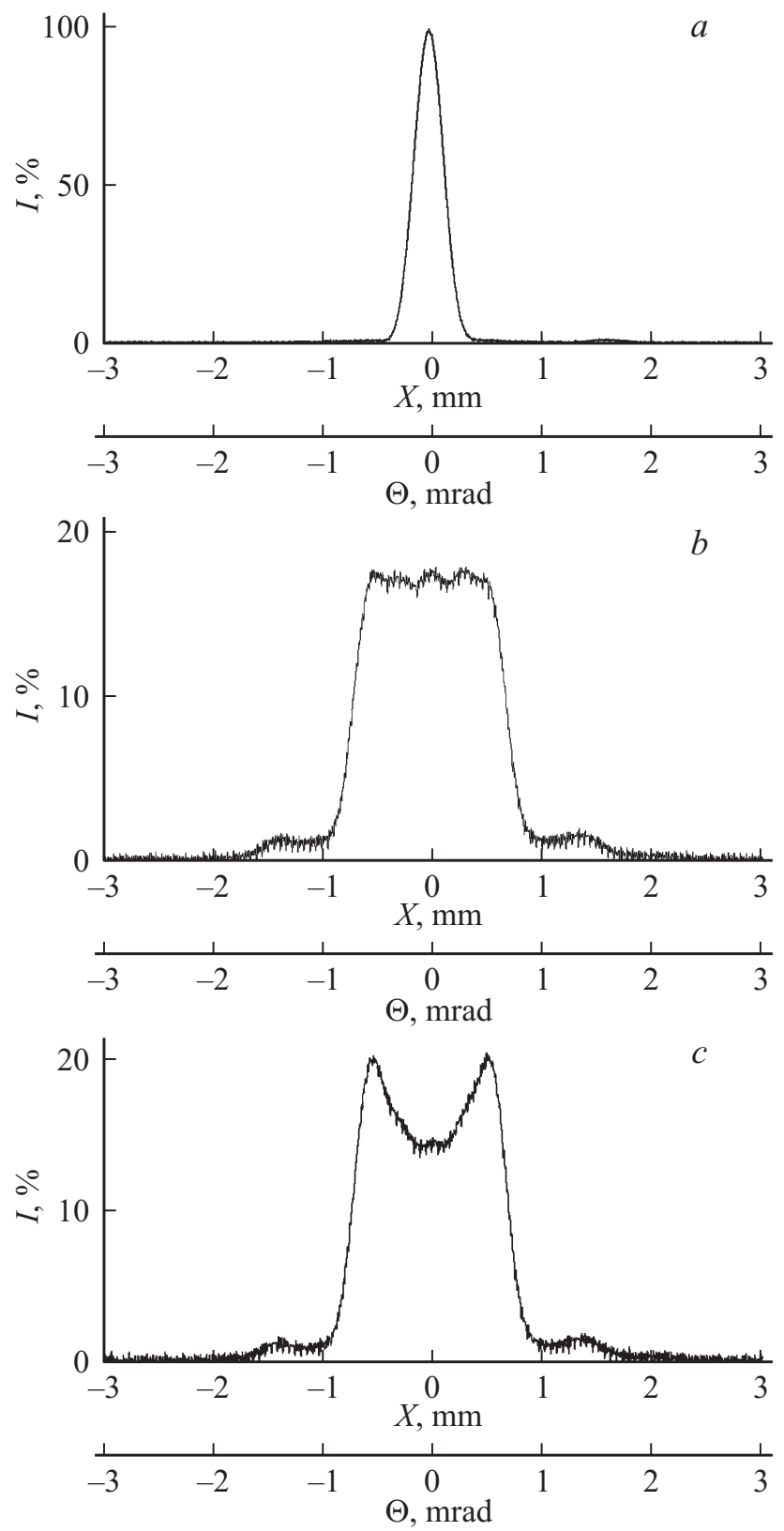

Рис. 7. Экспериментально измеренные профили лазерного луча: $a-$ исходный лазерный луч, $b-$ П-образный профиль, $c$ - профиль с провалом в центре.

BР104 с основными параметрами: скорость вращения щели $-10 \mathrm{~Hz}$, диаметр входного окна $-10 \mathrm{~mm}$, размер щели $10 \mu \mathrm{m}$. При расчете и создании оптической схемы выполнялось условие, чтобы $1 \mathrm{~mm}$ координатного смещения на измерителе профиля соответствовал $1 \mathrm{mrad}$ отклонения луча на выходе АО-модулятора. Оценочная относительная точность измерения положения луча составила $2-3 \%$.

На рис. 5: 1 - одномодовый лазер с длиной волны $0.63 \mu \mathrm{m}$ и апертурой $3 \mathrm{~mm}, 2$ - АО-дефлектор, 3 - линза с фокусом $1 \mathrm{~m}, 4$ - измеритель профиля лазерного луча.
$\mathrm{AO}$-дефлектор изготовлен из парателлурита $\left(\mathrm{TeO}_{2}\right)$, в котором возбуждалась медленная сдвиговая волна в направлении, составляющем $6^{\circ}$ с кристаллографической осью [110]. Исходный световой луч с длиной волны $1.06 \mu \mathrm{m}$ распространялся в кристалле под углом $\sim 3^{\circ}$ к оптической оси и имел поляризацию необыкновенной моды (так называемая геометрия взаимодействия широкополосного анизотропного дефлектора) [8].

Пьезопреобразователь выполнен из пластины ниобата лития $\left(\mathrm{LiNbO}_{3}\right)$ толщиной $60 \mu \mathrm{m}$, генерирующий чисто сдвиговую акустическую моду. Преобразователь обеспечивал эффективное преобразование ультразвука в полосе от 20 до $50 \mathrm{MHz}$, что было достигнуто использованием технологии акустического согласования импедансов [9].

Эксперименты проводились в ситуации, качественно аналогичной рис. 4, $a$, когда на АО-модулятор подавались пять радиочастот. В нашем случае разность частот была меньше для обеспечения перекрытия ближайших лучей: центральная частота $-41 \mathrm{MHz}$ и четыре симметрично расположенных боковых $\pm 0.3 \mathrm{MHz}, \pm 0.6 \mathrm{MHz}$. Итерационно подбирались амплитуды и фазы каждой частотной компоненты $\left(A_{n}\right.$ и $\left.\varphi_{n}\right)$ по критерию результата достижения П-образной формы луча при максимальной интенсивности. Как указывалось в (1), эта ситуация соответствует минимальной общей амплитудной модуляции управляющего электрического сигнала. Для иллюстрации последнего на рис. 6 показаны осциллограммы сигналов, подаваемых на АО-дефлектор.

Рис. 6, $a$ - временная зависимость амплитуды сигнала при произвольно установленных фазовых соотношениях между частотными компонентами. Рис. $6, b-$ то же, но фазовые соотношения подобраны по указанному критерию. Видно, что при неоптимальных фазах сигнал имеет значительную амплитудную модуляцию, а при подобранных соотношениях амплитудная модуляция существенно снижается (1).

На рис. 7 представлена серия экспериментально измеренных зависимостей относительной интенсивности ЛИ от сигналов управления. На графиках: $I$ - относительная интенсивность, $X$ - размер луча на измерителе профиля, $\Theta$ - пересчитанная угловая зависимость на выходе АО-дефлектора.

\section{Результаты эксперимента}

1. Многолучевая АО-дифракция позволяет реализовывать вид лазерного излучения, близкий к П-образному, при суммарной эффективности не менее чем 80-85\%.

2. Можно обеспечить и другие практически важные формы, например, реализовать ЭП с провалом в центре, что целесообразно при обработке металлов, когда нагрев в центре луча чрезмерен из-за отвода тепла.

Принципиальное достоинство метода - возможность быстропеременного изменения энергетической формы выходного луча. Быстродействие определяется скоростью звука в АО-ячейке и апертурой света и составило в эксперименте около $10 \mu \mathrm{s}$. 


\section{Выводы}

На базе многолучевой брегговской АО-дифракции предложено создание АО-шейпера. Детально рассмотрены условия, необходимые для реализации режима АОшейпера. В отличие от линзовых приборов такого типа акустооптические позволяют быстропеременно управлять формой оптического луча, меняя характер электрического управления. Быстрая перестройка формы луча может использоваться в технологических процессах лазерной обработки материалов для выбора оптимального технологического режима.

Проведены эксперименты на серийном АО-дефлекторе, подтвердившие расчетные результаты. Реализованы как П-образная энергетическая форма, так и форма с провалом в центре. Суммарные потери не превысили $80 \%$, быстродействие $10 \mu \mathrm{s}$.

Выражаем благодарность А.В. Вайнеру за внимание к работе.

\section{Список литературы}

[1] Магдич Л.Н., Молчанов В.Я. Акустооптические устройства и их применение. М.: Советское радио, 1978. 111 с.

[2] Электронный ресурс. Проспект фирмы Gooch \& Housego. Режим доступа: http://www.goochandhousego.com

[3] Донин В.И., Яковин Д.В., Грибанов А.В. // Письма в ЖТФ. 2015. Т. 101. Вып. 12. С. 881.

[4] Антонов С.Н. // ЖТФ. 2005. Т. 75. Вып. 4. С. 122.

[5] Антонов С.Н., Резвов Ю.Г. // ЖТФ. 2007. Т. 77. Вып. 8. C. 93.

[6] Антонов С.Н., Вайнер А.В., Проклов В.В., Резвов Ю.Г. // ЖТФ. 2008. Т. 78. Вып. 6. С. 79.

[7] Антонов С.Н., Вайнер А.В., Проклов В.В., Резвов Ю.Г. // ЖТФ. 2009. Т. 79. Вып. 11. С. 65.

[8] Семенков В.П. // ЖТФ. 1981. Т. 51. Вып. 10. С. 29.

[9] Антонов С.Н. // Акустический журнал. 2017. Т. 63. № 3. C. 1. 